\title{
Melatonin - More than Just a Pineal Hormone
}

\author{
Rüdiger Hardeland* \\ University of Goettingen, Germany
}

Received: August 30, 2017; Published: September 12, 2017

*Corresponding author: Rüdiger Hardeland, Johann Friedrich Blumenbach Institute of Zoology and Anthropology, University of Goettingen, Buergerstr 50, D-37073 Göttingen, Germany; Tel: +49551395414; Email: rhardel@gwdg.de

\begin{abstract}
Melatonin is not only synthesized in the pineal gland and the retina, but also in numerous other tissues. Extrapineal melatonin is formed in amounts exceeding by orders of magnitude that found in the pineal gland. This is particularly valid for the gastrointestinal tract, in which several physiological functions such as duodenal bicarbonate secretion have been elucidated. Melatonin is produced upon neuronal stimuli in enteroendocrine cells. Duodenal signaling involves the melatonin receptor $\mathrm{MT}_{2}$ present in enterocytes and in Paneth cells, in the latter case causing uroguanylin/guanylin release that leads to enterocytic activation of the receptor guanylyl cyclase GUCY2C. High amounts of melatonin are also found in the bile fluid, with contributions of melatonin synthesized in cholangiocytes and, presumably, enterohepatic circulation. Melatonin is also formed and metabolized in the skin, in which it contributes to photoprotection. In the cerebellum, melatonin formation is upregulated by bacterial lipopolysaccharide and conveys local anti-inflammatory protection. Increasing evidence indicates that melatonin does not easily cross membranes because of lipophilicity, but that transporters are required.
\end{abstract}

Keywords: Bile; Cerebellum; Gastrointestinal tract; Membranes; Skin

Abbreviations: CSF: Cerebrospinal Fluid; GIT: Gastrointestinal Tract; CFTR: Cystic Fibrosis Trans-membrane Conductance Regulator; PKC: Protein Kinase C; CaMK II: Calmodulin-Dependent Protein Kinase II; cGKII: Cyclic GMP-Dependent Protein Kinase II, AFMK: $N^{1}$-Acetyl- $N^{2}-$ formyl-5-methoxykynuramine; AMK: $N^{1}$-Acetyl-5-methoxykynuramine, AANAT: Aralkylamine $N$-Acetyltransferse

\section{Introduction}

Numerous publications and manuscripts prior to review start with the incorrect statement that "melatonin is mainly synthesized in the pineal gland". Meanwhile, authors have perceived that melatonin is also formed in extrapineal sites. However, the amounts of extrapineally formed melatonin are frequently strongly underrated. A correct statement would be that, in mammals and under physiological conditions, circulating melatonin is mainly released from the pineal gland. This organ also secretes melatonin, via the pineal recess, into the third ventricle of the brain [1-3]. The chronobiological actions of mammalian melatonin are widely based on its nocturnal release into the blood and the cerebrospinal fluid (CSF). Insofar pineal melatonin is, in mammals, chronobiologically privileged, whereas in nonmammalian vertebrates, the contribution by other sites, especially the retina, can be much more important. Even in mammals including the human, the amounts of melatonin released from an extrapineal site can exceed, after pharmacological treatment, the levels generated by the pineal gland. After a high tryptophan load, melatonin is released, already during the day, by the gastrointestinal tract in quantities much above concentrations attained during maximal pineal secretion $[4,5]$. These findings are known since a quarter of a century, but widely neglected by many researchers.
Meanwhile, numerous other extrapineal sites of melatonin formation have been identified [6,7]. Moreover, the exclusive role of melatonin as a hormone in the strict, traditional sense had to be expanded to paracrine and autocrine actions [8]. However, this does not fundamentally differ from other endocrine factors and rather reflects a modern, more correct view on that what we call a hormone, to which earlier definitions have turned out to be inappropriate. It is the aim of this mini review to sharpen the awareness of researchers towards the multiple sites of melatonin formation and the consequences thereof.

\section{Extrapineal sites and the problem of poor melatonin release}

As summarized elsewhere [6,7], melatonin formation has been demonstrated in various regions of the central nervous system, especially cerebellum and retina, in the membranous cochlea, lens, glomus caroticum, gastrointestinal tract (GIT), cholangiocytes of liver, bone marrow, various leukocytes, thymus, spleen, kidney, heart, ovary, placenta, skin, airway epithelia, and, in rodents, the Harderian gland. The amounts formed there are highly different and, with regard to the brain, divergent [9]. In many cases studied, with the exception of the retina, circadian rhythmicity of extrapineal 
melatonin only displays low amplitudes or is virtually absent [6]. In the GIT, a low-amplitude rhythm was demonstrable in some species, but was poorly detectable in others. In rats, a rhythm of duodenal melatonin preceded that of the pineal gland, indicating some autonomy from the pineal gland $[10,11]$.

The high quantities of releasable GIT melatonin have been indicative of substantial amounts present in this organ system. In fact, calculations of overall GIT melatonin led to the conclusion that it exceeds that present in the pineal gland by about 400 to 500 times [12,13]. Of course, this is less a matter of concentration rather than of the much larger size of the GIT. However, the high amounts present in the extrapineal sites of melatonin formation raise an important question, and the answer has the potential of changing our view on the physical properties of melatonin. The problem concerns the reason for why so little of melatonin is released from the extrapineal sources, except for extraordinary conditions of the afore mentioned tryptophan load. This problem had been addressed some years ago [14]. One can read in many publications that melatonin crosses membranes with ease. Sometimes, it is also erroneously stated that melatonin were a highly lipophilic substance, which is certainly not the case.

Instead, melatonin is better described as amphiphilic [14]. If melatonin should be really able to easily cross membranes because of its physical properties of solubility, it would be hard to understand why it is so poorly released from the extrapineal sites. If the solubility properties were correct, the only possible explanation would be sequestration by binding proteins, molecules that should, however, be different from receptors [14]. The only alternative would be that the interpretation that had led to the assumption of easy membrane crossing is simply wrong. Actually, this latter possibility may be preferable.

\section{A few examples of the roles of extrapineal melatonin}

A full record of all reports on extrapineal melatonin would exceed the scope of this mini review. Many details can be found elsewhere in comprehensive reviews [6,7]. Instead a few examples shall illustrate that substantial progress has been made in the understanding of melatonin's functions beyond those from the pineal source. The focus will be on physiological and cell biological regulation mechanisms, and not primarily on scavenging by free radicals, an effect that may exist in any cell that has formed or accumulated sufficiently high concentrations of melatonin.

The first example concerns the GIT. After earlier reports on melatonin synthesis in enteroendocrine (enterochromaffin) cells (EC cells) previously summarized [6], some functions were identified and analyzed, such as bicarbonate and water release to the lumen [13] as well as modulation of motility $[6,13]$. Meanwhile, the control of bicarbonate secretion has been largely elucidated $[15,16]$. EC cells are stimulated to produce melatonin by the autonomous nervous system in two ways:

(i) by norepinephrine release from sympathetic fibers and action via $\alpha^{1}$-adrenergic receptors

(ii) by acetylcholine released from postganglionic fibers originating at ganglia of the enteral nervous system.
These ganglia may be stimulated by either sympathetic or parasympathetic fibers, while the postganglionic acetylcholine acts via muscarinic receptors at the EC cells. Melatonin from the EC cells acts via the melatonin receptor $\mathrm{MT}_{2}$ at both enterocytes and Paneth cells. In enterocytes, $\mathrm{MT}_{2}$ signaling leads to the activation of phospholipase C (PLC) and, therefore, ultimately to increases in cytosolic $\mathrm{Ca}^{2+}$, which directly acts at the bicarbonate-releasing channel CFTR. For mechanistic reasons, a concomitant chloride release via the same channel and an associated water release can be assumed. Stimulation of PLC also causes activation of protein kinase C (PKC) and calmodulin-dependent protein kinase II (CaMK II). These enzymes may further enhance CFTR opening, which would require further experimental support. However, another protein kinase generates such an effect. $\mathrm{MT}_{2}$ signaling causes Paneth cells to release uroguanylin and/or guanylin, which are ligands at the receptor GUCY2C, the membrane-associated guanylyl cyclase $2 \mathrm{C}$ present in the enterocytic plasma membrane. Cyclic GMP formed by GUCY2C activates cGKII (cyclic GMP-dependent protein kinase II), which phosphorylates CFTR and thereby, enhances bicarbonate release [16]. Other effects of melatonin in the GIT concern stimulation of duodenal cholecystokinin secretion and enhancement of mucosal blood flow in the tubular GIT [13]. However, these actions would require further analysis in terms of signaling mechanisms.

Melatonin formation has been also described for hepatic cholangiocytes [7]. From there, melatonin is released into the bile, a fluid that contains in humans and other mammals several thousand $\mathrm{pg} / \mathrm{mL}$ at daytime $[17,18]$, much above the serum daytime levels that usually range between 3 and $20 \mathrm{pg} / \mathrm{mL}$. What the function of these high levels really is, apart from the usually assumed protective role, remains to be clarified. For instance, this might concern a further stimulation of duodenal bicarbonate and water release. Moreover, the question has been addressed whether the high concentrations are due to the synthetic capacity of cholangiocytes, which seems to be rather unlikely. Instead, it has been assumed that elevated biliary melatonin levels may be a consequence of enterohepatic recirculation [18].

Another melatonin-producing organ that has attracted increasing attention is the skin. The enzymes of melatonin synthesis had been detected in this organ more than a decade ago $[19,20]$. Meanwhile, melatonin measurements have been conducted in the human skin and the locally formed metabolites, 6-hydroxymelatonin, 5-methoxytryptamine, $N^{1}$-acetyl- $N^{2}$-formyl-5-methoxykynuramine (AFMK), and $N^{1}$-acetyl-5-methoxykynuramine (AMK) have been determined [21,22]. The concentrations of the indolic metabolites exceeded by far that of melatonin. Interestingly, Afro-Americans had higher concentrations of all these compounds than Caucasians, a finding indicative of a protective role of melatonin in this organ that is exposed to radiation and other external noxes. The assumed protection [23] has recently received support by exposing test persons to UV sunlight with or without topical application of a melatonin containing cream, thereby demonstrating the prevention of erythema [24,25]. Additional physiological and protective actions of melatonin are likely. 
As another example of an extrapineal source of melatonin, the cerebellum shall be briefly discussed. In this part of the CNS, the expression of aralkylamine $N$-acetyltransferse (AANAT), the rate-limiting enzyme of melatonin formation, and the presence of melatonin have been known since long [26,27]. However, it had remained unclear whether melatonin is formed in this place or taken up and whether AANAT expression is mainly used there for formation of $\mathrm{N}$-acetylserotonin, which has properties beyond the role as a melatonin precursor. More recently, it had been shown that melatonin formation really takes place in the cerebellum and is upregulated upon exposure to bacterial lipopolysaccharideinduced oxidative stress [28]. The rise in cerebellar melatonin was not prevented by pinealectomy and, thus, did not result from increased melatonin release via the pineal recess. No similar effects were observed in the cortex and in the hippocampus. Moreover, the increase in cerebellar melatonin did not exert any protective effects in these other brain regions. Again, this is indicative of a local persistence of melatonin with no substantial release from the site of formation.

\section{Conclusion}

Concerning melatonin, there are several messages that should be divulgated within the community. First, melatonin is not only produced in the pineal gland and the quantities of extrapineal melatonin are by orders of magnitude higher than in the pineal. Therefore, statements that melatonin is mainly produced in the pineal gland are incorrect. In mammals, the release of melatonin from extrapineal sites is, under normal conditions, usually rather low. However, after a high tryptophan load, supraphysiological levels of blood melatonin can be attained by release from the GIT. Concentrations much above serum levels are also present in the bile fluid, a phenomenon to which melatonin synthesis in cholangiocytes and, presumably, enterohepatic circulation contribute. Melatonin is formed and metabolized in the skin, in which substantial amounts of the parent compound as well as of its indolic and kynuric metabolites are demonstrable.

Cutaneous melatonin is obviously involved in photoprotection and may play additional physiological and protective roles. Melatonin is also formed in several areas of the CNS. It has been shown to be upregulated in the cerebellum upon a challenge by bacterial lipopolysaccharide. It conveys local protection to the cerebellum, but not to other brain areas. Evidence is increasing that melatonin does not easily cross membranes because of lipophilicity, contrary to earlier belief. Anyway, melatonin is amphiphilic rather than lipophilic, especially not highly lipophilic, what can be frequently read despite its incorrectness. It seems that melatonin penetration through membranes is a process controlled by transporters. Recently, this view has been supported by findings on the involvement of oligopeptide transporters PEPT 1/2 and the organic anion transporter OAT3, which may play a role in the crossing of the plasma membrane as well as intracellular, especially mitochondrial membranes $[29,30]$. With regard to mitochondria, these findings may be of particular importance, because of the observed accumulation of melatonin in these organelles [31,32] and intramitochondrial signaling by the melatonin receptor $\mathrm{MT}_{1}$ [33].

\section{References}

1. Tricoire H, Locatelli A, Chemineau P, Malpaux B (2002) Melatonin enters the cerebrospinal fluid through the pineal recess. Endocrinology 143(1): 84-90.

2. Reiter RJ, Tan DX, Kim SJ, Cruz MH (2014) Delivery of pineal melatonin to the brain and SCN: role of canaliculi, cerebrospinal fluid, tanycytes and Virchow-Robin perivascular spaces. Brain Struct Funct 219(6): 18731887.

3. Tan DX, Manchester LC, Reiter RJ (2016) CSF generation by pineal gland results in a robust melatonin circadian rhythm in the third ventricle as an unique light/dark signal. Med Hypotheses 86: 3-9.

4. Hajak G, Huether G, Blanke J, Blömer M, Freyer C, et al. (1991) The influence of intravenous L-tryptophan on plasma melatonin and sleep in men. Pharmacopsychiatry 24(1): 17-20.

5. Huether G, Poeggeler B, Reimer A, George A (1992) Effect of tryptophan administration on circulating melatonin levels in chicks and rats: evidence for stimulation of melatonin synthesis and release in the gastrointestinal tract. Life Sci (12): 945-953.

6. Hardeland R, Cardinali DP, Srinivasan V, Spence DW, Brown GM, et al. (2011) Melatonin - A pleiotropic, orchestrating regulator molecule. Prog Neurobiol 93(3): 350-384.

7. Acuña Castroviejo D, Escames G, Venegas C, Díaz-Casado ME, Lima Cabello E, et al. (2014) Extrapineal melatonin: sources, regulation, and potential functions. Cell Mol Life Sci 71(16): 2997-3025.

8. Tan DX, Manchester LC, Hardeland R, Lopez Burillo S, Mayo JC, et al. (2003) Melatonin - a hormone, a tissue factor, an autocoid, a paracoid, and an antioxidant vitamin. J Pineal Res 34(1): 75-78.

9. Hardeland R (2010) Melatonin metabolism in the central nervous system. Curr Neuropharmacol 8(3): 168-181.

10. Zeman M, Józsa R, Cornélissen G, Stebelova K, Bubenik G, et al. (2005) Chronomics: Circadian lead of extrapineal vs. pineal melatonin rhythms with an infradian hypothalamic exploration. Biomed Pharmacother 59(Suppl 1): S213-S219.

11. Poeggeler B, Cornélissen G, Huether G, Hardeland R, Józsa R, et al. (2005) Chronomics affirm extending scope of lead in phase of duodenal vs. pineal circadian melatonin rhythms. Biomed Pharmacother 59(Suppl 1): S220-S224.

12. Huether G (1993) The contribution of extrapineal sites of melatonin synthesis to circulating melatonin levels in higher vertebrates. Experientia 49(8): 665-670.

13. Bubenik GA (2002) Gastrointestinal melatonin: localization, function, and clinical relevance. Dig Dis Sci 47(10): 2336-2348.

14. Hardeland R, Poeggeler B (2008) Melatonin beyond its classical functions. Open Physiol J 1: 1-23.

15. Flemström G, Sjöblom M (2005) Epithelial cells and their neighbors. II. New perspectives on efferent signaling between brain, neuroendocrine cells, and gut epithelial cells. Am J Physiol Gastrointest Liver Physiol 289(3): G377-G380.

16. Bengtsson MW, Jedstedt G, Flemström G (2007) Duodenal bicarbonate secretion in rats: stimulation by intra-arterial and luminal guanylin and uroguanylin. Acta Physiol (Oxf) 191(4): 309-317.

17. Tan D, Manchester LC, Reiter RJ, Qi W, Hanes MA, et al. (1999) High physiological levels of melatonin in the bile of mammals. Life Sci 65(23): 2523-2529.

18. Reiter RJ, Rosales Corral SA, Manchester LC, Liu X, Tan DX (2014) Melatonin in the biliary tract and liver: health implications. Curr Pharm Des 20(30): 4788-4801.

19. Slominski A, Pisarchik A, Semak I, Sweatman T, Wortsman J, et al. (2002) Serotoninergic and melatoninergic systems are fully expressed in human skin. FASEB J 16(8): 896-898. 
20. Slominski A, Fischer TW, Zmijewski MA, Wortsman J, Semak I, et al. (2005) On the role of melatonin in skin physiology and pathology. Endocrine 27(2): 137-148.

21. Kim TK, Lin Z, Tidwell WJ, Li W, Slominski AT (2015) Melatonin and its metabolites accumulate in the human epidermis in vivo and inhibit proliferation and tyrosinase activity in epidermal melanocytes in vitro. Mol Cell Endocrinol 404: 1-8.

22. Kim TK, Lin Z, Li W, Reiter RJ, Slominski AT (2015) N1-Acetyl-5methoxykynuramine (AMK) is produced in the human epidermis and shows antiproliferative effects. Endocrinology 156(5): 1630-1636.

23. Slominski AT, Kleszczyński K, Semak I, Janjetovic Z, Zmijewski MA, et al. (2014) Local melatoninergic system as the protector of skin integrity. Int J MolSci 15(10): 17705-17732.

24. Scheuer C, Pommergaard HC, Rosenberg J, Gögenur I (2016) Dose dependent sun protective effect of topical melatonin: A randomized placebo-controlled, double-blind study. J Dermatol Sci 84(2): 178-185.

25. Scheuer C (2017) Melatonin for prevention of erythema and oxidative stress in response to ultraviolet radiation. Dan Med J 64(6): B5358.

26. Uz T, Qu T, Sugaya K, Manev H (2002) Neuronal expression of arylalkylamine $\mathrm{N}$-acetyltransferase (AANAT) mRNA in the rat brain. Neurosci Res 42(2): 309-316.
27. Hardeland R, Poeggeler B (2012) Melatonin and synthetic melatonergic agonists: actions and metabolism in the central nervous system. Cent Nerv Syst Agents Med Chem 12(3): 189-216.

28. Pinato L, da Silveira Cruz Machado S, Franco DG, Campos LM, Cecon E, et al. (2015) Selective protection of the cerebellum against intracerebroventricular LPS is mediated by local melatonin synthesis. Brain Struct Funct 220(2): 827-840.

29. Huo X, Wang C, Yu Z, Peng Y, Wang S, et al. (2017) Human transporters, PEPT1/2, facilitate melatonin transportation into mitochondria of cancer cells: An implication of the therapeutic potential. J Pineal Res $62(4)$.

30. Mayo JC, Sainz RM, González Menéndez P, Hevia D, Cernuda Cernuda R (2017) Melatonin transport into mitochondria. Cell Mol Life Sci

31. Messner M, Hardeland R, Rodenbeck A, Huether G (1998) Tissue retention and subcellular distribution of continuously infused melatonin in rats under near physiological conditions. J Pineal Res 25(4): 251-259.

32. López A, García JA, Escames G, Venegas C, Ortíz F, et al. (2009) Melatonin protects the mitochondria from oxidative damage reducing oxygen consumption, membrane potential, and superoxide anion production. J Pineal Res 46(2):188-198.

33. Hardeland R (2017) Melatonin and the electron transport chain. Cell Mol Life Sci, [epub ahead of print, Aug 7]; doi: 10.1007/s00018-017-2615-9.

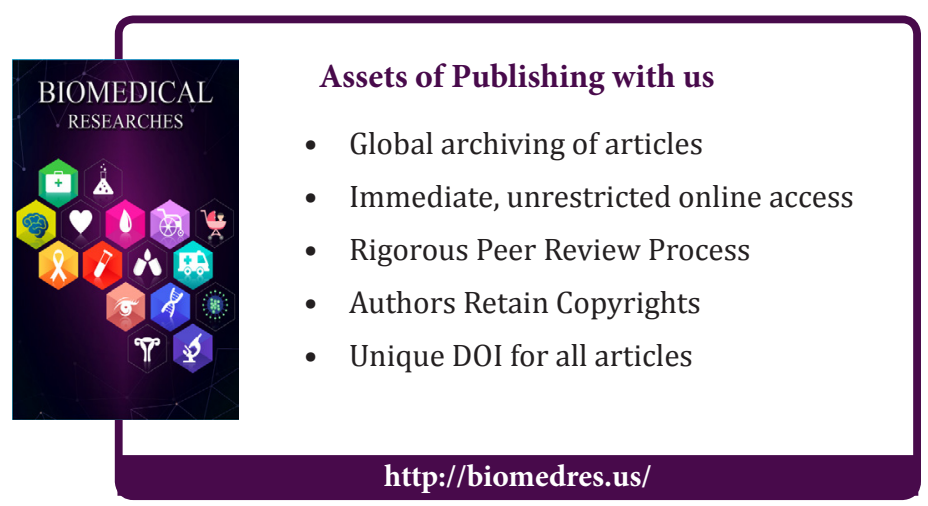

